\title{
Genome analysis of Pseudoalteromonas flavipulchra JG1 reveals various survival advantages in marine environment
}

\author{
Min Yu', Kaihao Tang ${ }^{1}$, Jiwen Liu', Xiaochong Shi', Tobias AM Gulder ${ }^{2}$ and Xiao-Hua Zhang ${ }^{1,3^{*}}$
}

\begin{abstract}
Background: Competition between bacteria for habitat and resources is very common in the natural environment and is considered to be a selective force for survival. Many strains of the genus Pseudoalteromonas were confirmed to produce bioactive compounds that provide those advantages over their competitors. In our previous study, P. flavipulchra JG1 was found to synthesize a Pseudoalteromonas flavipulchra antibacterial Protein (PfaP) with L-amino acid oxidase activity and five small chemical compounds, which were the main competitive agents of the strain. In addition, the genome of this bacterium has been previously sequenced as Whole Genome Shotgun project (PMID: 22740664). In this study, more extensive genomic analysis was performed to identify specific genes or gene clusters which related to its competitive feature, and further experiments were carried out to confirm the physiological roles of these genes when competing with other microorganisms in marine environment.

Results: The antibacterial protein PfaP may also participate in the biosynthesis of 6-bromoindolyl-3-acetic acid, indicating a synergistic effect between the antibacterial macromolecule and small molecules. Chitinases and quorum quenching enzymes present in $P$. flavipulchra, which coincide with great chitinase and acyl homoserine lactones degrading activities of strain JG1, suggest other potential mechanisms contribute to antibacterial/antifungal activities. Moreover, movability and rapid response mechanisms to phosphorus starvation and other stresses, such as antibiotic, oxidative and heavy metal stress, enable JG1 to adapt to deleterious, fluctuating and oligotrophic marine environments.

Conclusions: The genome of $P$. flavipulchra JG1 exhibits significant genetic advantages against other microorganisms, encoding antimicrobial agents as well as abilities to adapt to various adverse environments. Genes involved in synthesis of various antimicrobial substances enriches the antagonistic mechanisms of P. flavipulchra JG1 and affords several admissible biocontrol procedures in aquaculture. Furthermore, JG1 also evolves a range of mechanisms adapting the adverse marine environment or multidrug rearing conditions. The analysis of the genome of $P$. flavipulchra JG1 provides a better understanding of its competitive properties and also an extensive application prospect.
\end{abstract}

Keywords: Pseudoalteromonas flavipulchra, Genome analysis, Antibacterial metabolites, Quorum quenching, Survival advantages

\footnotetext{
* Correspondence: xhzhang@ouc.edu.cn

${ }^{1}$ College of Marine Life Sciences, Ocean University of China, Qingdao 266003,

China

${ }^{3}$ Mailing address: College of Marine Life Sciences, Ocean University of China

5 Yushan Road, Qingdao 266003, China

Full list of author information is available at the end of the article
} 


\section{Background}

The genus Pseudoalteromonas was established in 1995 and was found to be ubiquitous in the marine environment [1]. Numerous Pseudoalteromonas strains were isolated from the polar region, inshore waters or surfaces of marine organism, and were shown to synthesize a range of bioactive molecules [2-4]. The production of molecules that are active against a variety of target organisms appears to be an important characteristic for this genus and may greatly benefit Pseudoalteromonas cells in their competition for nutrients or colonization of surfaces [5]. P. tunicata D2, as a model organism to study, was demonstrated to synthesize a range of inhibitory substances, including a toxic antibiotic protein and two pigments [6,7]. Analysis of its complete genome sequence revealed that several genes and gene clusters were involved in the production of inhibitory compounds that were associated with its successful persistence and competition on marine surfaces [8].

P. flavipulchra JG1 was isolated from rearing water of healthy turbot (Scophthalmus maximus) in Qingdao, China. Strain JG1 is capable of adapting to the oligotrophic marine environment and reveals various advantageous survival abilities among competitive species. It was demonstrated to exhibit inhibitory activity against many Vibrio, Aeromonas and Bacillus strains and was nontoxic to zebra fish and mantis shrimp [9]. Furthermore, Strain JG1 was shown to synthesize the putative L-amino acid oxidase named Pseudoalteromonas flavipulchra antibacterial Protein (PfaP) and 5 small molecular compounds with antibacterial activity. These compounds were identified as $p$-hydroxybenzoic acid, trans-cinnamic acid, 6bromoindolyl-3-acetic acid, $N$-hydroxybenzoisoxazolone and 2 -deoxyadenosine. All of these metabolites have been observed to exhibit antibacterial activities against several pathogens, including $V$. anguillarum, $V$. harveyi, Photobacterium damselae subsp. damselae and A. hydrophila [10]. The inhibitory properties of P. flavipulchra JG1 against fish pathogens indicate that the strain or its products could be utilized as biocontrol agent(s) in aquaculture.
To obtain a better understanding of the genetic potential of P. flavipulchra JG1 as a biocontrol organism, we have sequenced and analyzed its genome and compared it to the genomic data of closely related strains publicly available. We have found that the P. flavipulchra genome contains several genes and gene clusters that might be involved in the production of inhibitory compounds against pathogens and competitors in the marine environment. The analysis of P. flavipulchra genome also verifies excellent capabilities of this strain to adapt to environmental changes and challenges.

\section{Results and discussion}

\section{Genome features and comparison with other Pseudoalteromonas genomes}

The P. flavipulchra JG1 genome is composed of 5565 $361 \mathrm{bp}$ and the calculated $\mathrm{G}+\mathrm{C}$ content is $43.23 \%$. A total of 4913 open reading frames (ORFs) are identified within the $P$. flavipulchra JG1 genome (Table 1). Among the predicted genes, $1985(40.4 \%)$ are not found in COG categories, and 1725 (35.1\%), 712 (14.5\%), 1863 (37.9\%) and 629 (12.8\%) genes are not applicable within the KEGG, NR, SwissProt and TrEMBL databases, respectively [11]. JG1 contains 180 tandem repeat regions, 143 transposons, and 5 ISs (Insert sequences) that account for about $1.1 \%$ of the genome. The P. flavipulchra JG1 genome is larger than that of the five other Pseudoalteromonas strains whose genomic sequences have been published in the IMG database: P. tunicata D2 (Gi05080), P. haloplanktis TAC125 (Gc00289), P. atlantica T6c (Gc00395) and two Pseudoalteromonas strains TW-7 (Gi01432) and SM9913 (Gc01563). D2 is an antibiofouling bacterium, both T6c and TW-7 are mesophile bacteria, and TAC125 and SM9913 are psychrotolerant adapting to cold aqueous and deep-sea sediment environment, respectively. General features of these genomes were given in Table 1 and their phylogenetic relationship based on the 16S rRNA gene (Figure 1) indicated that $P$. flavipulchra JG1 formed a cluster with P. tunicata D2. Comparing with other sequenced Pseudoalteromonas strains, P. tunicata D2 [12] also exhibited great inhibitory

Table 1 General features of JG1 and other Pseudoalteromonas genomes

\begin{tabular}{|c|c|c|c|c|c|c|}
\hline & P. flavipulchra JG1 & P. tunicata D2 & P. atlantica T6c & $\begin{array}{l}\text { P. haloplanktis } \\
\text { TAC } 125\end{array}$ & $\begin{array}{c}\text { Pseudoalteromonas } \\
\text { sp. TW-7 }\end{array}$ & $\begin{array}{c}\text { Pseudoalteromonas } \\
\text { sp. SM9913 }\end{array}$ \\
\hline Genome size & 5505361 & 4982425 & 5187005 & 3850272 & 4104952 & 4037671 \\
\hline CDS number & 4913 & 4505 & 4313 & 3487 & 3783 & 3712 \\
\hline CDS length & 4828917 & 4421622 & 4507935 & 3404858 & 3673686 & 3569827 \\
\hline $\mathrm{G}+\mathrm{C}$ percentage & $43 \%$ & $40 \%$ & $45 \%$ & $40 \%$ & $40 \%$ & $40 \%$ \\
\hline RNA number & 158 & 133 & 92 & 147 & 94 & 87 \\
\hline CDS assigned to COG & 3314 & 3096 & 3279 & 2639 & 2797 & 2860 \\
\hline COG Cluster number & 1801 & 1791 & 1846 & 1731 & 1778 & 1768 \\
\hline
\end{tabular}




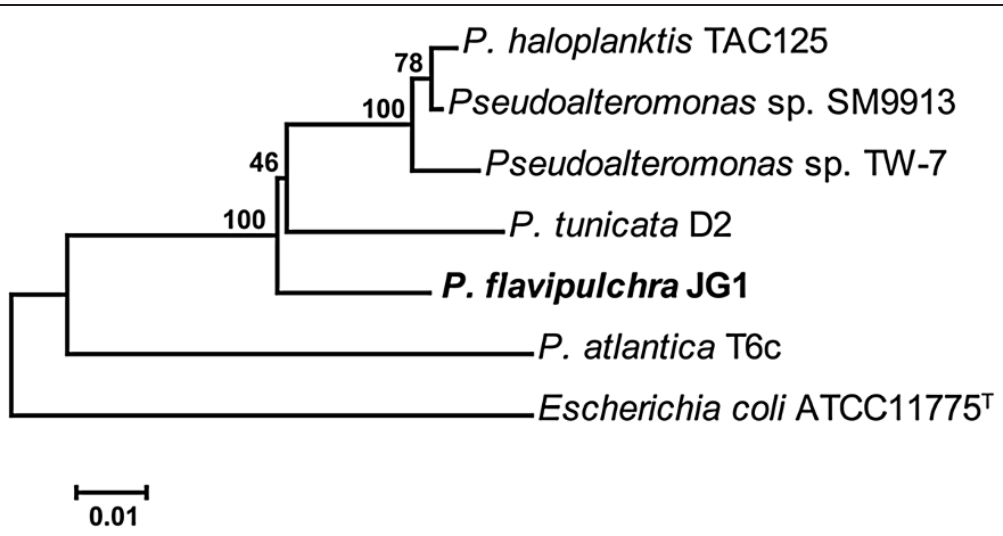

Figure 1 Phylogenetic tree based on 16S rDNA of JG1 and Pseudoalteromonas strains compared in the study. Tree was generated by Neighbor-Joining method with 1000 bootstrap replications. The sequence alignment and phylogenetic calculations were performed with MEGA 5.

activities against several specific organisms as in $P$. flavipulchra JG1 [10], indicating that P. flavipulchra was functionally closely related to $P$. tunicata D2.

P. flavipulchra JG1, P. tunicata D2 and P. haloplanktis TAC125 share 1898 orthologous genes, accounting for $38.63 \%, 42.14 \%$ and $54.47 \%$ of all genes of JG1, D2 and TAC125, respectively. In addition, JG1 contains more specific genes $(2248,45.76 \%)$ than D2 (1856, 41.21\%) and TAC125 (1067, 30.63\%), and there are more common genes within JG1 and D2 (Figure 2A). We have also conducted a COG-based analysis among the specific and orthologous genes of these three strains. As shown in Figure 2B, a larger proportion of JG1 and D2 specific genes belong to signal transduction mechanisms $(\mathrm{T})$, while the genome of TAC125 harbors more specific genes belonging to lipid transport and metabolism (I). Genes involved in signal transduction were considered to allow $P$. tunicata D2 to generate phenotypic variation and provide the capacity of niche adaptation [8], and genes assigned to the same COG category might also play a similar role in P. flavipulchra JG1 in that both D2 and JG1 exhibited competitive activities in their surrounding environments. Contrasting, in order to adapt cold condition, TAC125 needs more genes involved in lipid transport and metabolism such as genes coding for lipid desaturase, which could increase membrane fluidity at low temperature [13]. According to the genomic comparison results, JG1 and D2 share more functional similarities than to TAC125.

Significantly, P. flavipulchra contains the highest proportion of specific genes belonging to the COG categories defense mechanisms (V) and secondary metabolites biosynthesis, transport and catabolism (Q), which account for $4.56 \%$ and $5.40 \%$, respectively, among other bacteria. (Additional file 1: Figure S1). This mainly attributes to more genes involved in $\mathrm{ABC}$-type antimicrobial peptide transport system (COG0577, COG1136), beta- lactamase class $\mathrm{C}$ and other penicillin binding proteins (COG1680), cation/multidrug efflux pump (COG0841), ABC-type siderophore export system (COG 4615) and those related to the synthesis of potential bioactive compounds, such as nonribosomal peptide synthetase (NRPS) modules (COG1020) and polyketide synthases (COG 3321). The abundance of genes involved in expression and transport of potential primary and secondary metabolites and defense compounds are consistent with the capability of P. flavipulchra to produce various antimicrobial compounds [10] and generate survival advantages in marine environments [8].

\section{Biosynthesis of antimicrobial metabolites}

P. flavipulchra JG1 was demonstrated to synthesize the antibacterial protein PfaP and 5 known small molecular compounds with antibacterial activity against $V$. anguillarum [10].

The antibacterial protein $\mathrm{PfaP}$ is a homologue of L-amino acid oxidase with high similarity to the L-lysine oxidase AlpP of P. tunicata D2 (GenBank AAP73876.1) [6] and marinocine antimicrobial protein of Marinomonas mediterranea MMB-1 (GenBank AAY33849.1) [14]. The sequence identities of protein $\mathrm{PfaP}$ and these two antimicrobial proteins were $58 \%$ and $54 \%$, with coverages of $95 \%$ and $92 \%$, respectively. PfaP is a secreted protein that might catalyze the oxidative deamination of specific amino acids to the respective $\alpha$-keto acids with concomitant release of ammonium and hydrogen peroxide [15]. The antibacterial activity of extracellular proteins of JG1 could be abolished in the presence of catalase, suggesting that the inhibitory effect was directly mediated by the action of hydrogen peroxide (Figure 3). The gene located downstream of $p f a P$ (FaGL1049) codes a catalase (FaGL1053) which responds to oxidative stress and causes decomposition of hydrogen peroxide. The catalase activity might thus immediately protect JG1 from the damage of the 
A
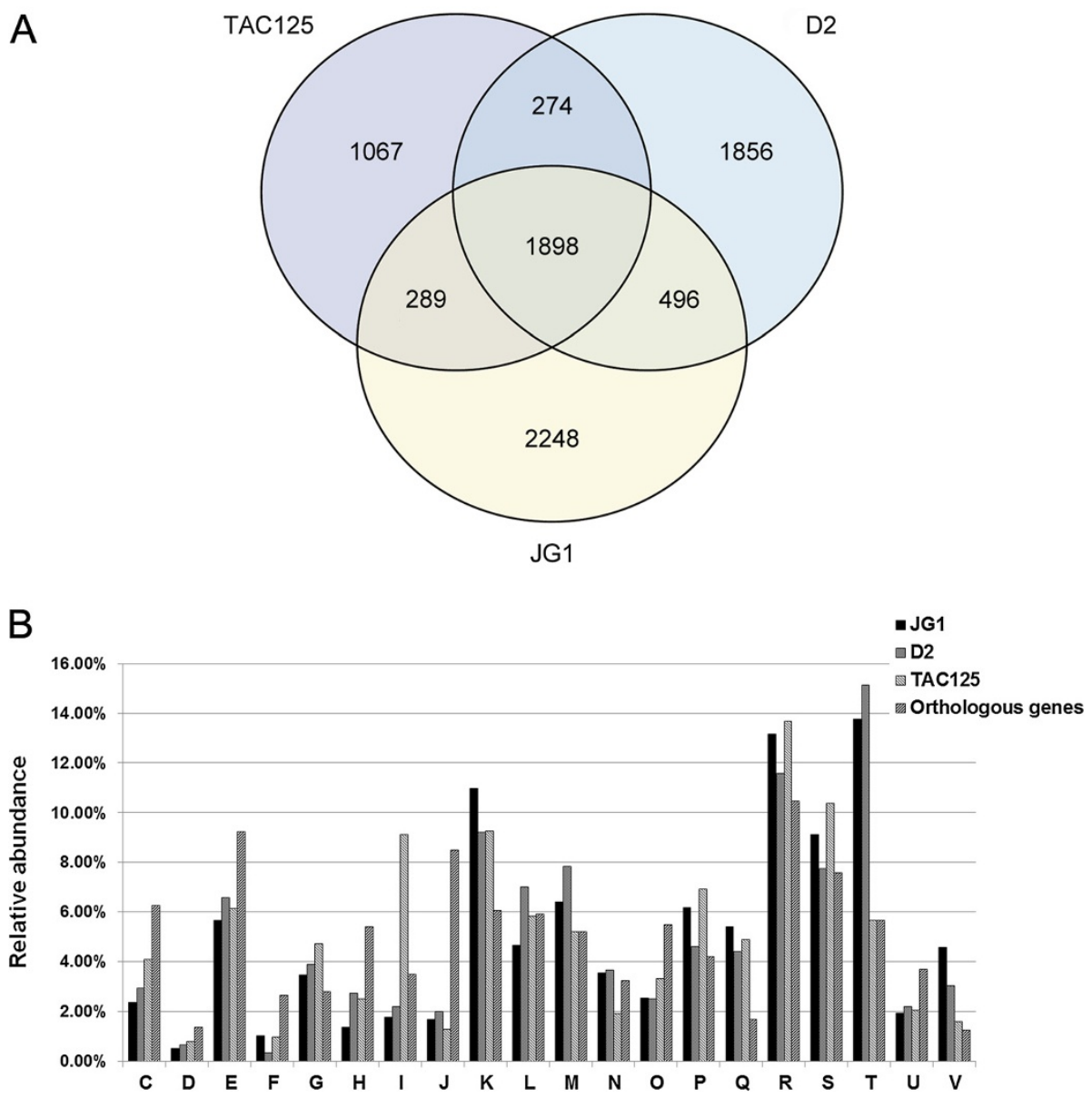

Figure 2 Comparison of the gene content of $P$. flavipulchra JG1, P. tunicata D2 and P. haloplanktis TAC125. Putative orthologous genes are defined as reciprocal best hit proteins with a minimum $40 \%$ identity and $70 \%$ of the length of the query protein, calculated by the BLAST algorithm. (A) Venn diagram of the orthologous and specific genes in each strain. (B) Relative abundance compared to all COG categories of the orthologous and specific genes in each strain. COG functional categories are described as follows: C, energy production and conversion; D, cell cycle control, cell division, chromosome partitioning; E, amino acid transport and metabolism; F, nucleotide transport and metabolism; G, carbohydrate transport and metabolism; $\mathrm{H}$, coenzyme transport and metabolism; I, lipid transport and metabolism; J, translation, ribosomal structure and biogenesis; $\mathrm{K}$, transcription; L, replication, recombination and repair; M, cell wall/membrane/envelope biogenesis; $\mathrm{N}$, cell motility; $\mathrm{O}$, posttranslational modification, protein turnover, chaperones; P, inorganic ion transport and metabolism; Q, secondary metabolites biosynthesis, transport and catabolism; R, general function prediction only; S, function unknown; $T$, signal transduction mechanisms; $\mathrm{U}$, intracellular trafficking, secretion, and vesicular transport; $V$, defense mechanisms.

hydrogen peroxide generated by PfaP. We also identified 3 further genes encoding catalases and several genes capable of hydrogen peroxide degradation in the genome. Thus, JG1 produces the antimicrobial protein PfaP and simultaneously possesses several genes related to potential selfprotection mechanisms.

The compounds $p$-hydroxybenzoic acid, trans-cinnamic acid, and 6-bromoindolyl-3-acetic acid isolated from P. flavipulchra JG1 were demonstrated to inhibit the growth of several pathogens [10]. We now identified several genes most likely involved in the biosynthesis of these metabolites.

Compound $p$-hydroxybenzoic acid and its derivatives are widely applied as food preservatives and stabilizers (antioxidants). As an organic acid, it has also been shown to inhibit the growth of ethanologenic Escherichia coli LY01 and ethanol synthesis [16]. The compound is probably derived from chorismate by action of a chorismate lyase, resembling the first step of ubiquinone biosynthesis. $p$-hydroxybenzoic acid is an important precursor of ubiquinones and $P$. flavipulchra harbors the ubiCA gene cluster (FaGL1386 and FaGL1387) responsible for the conversion of chorismate to $p$-hydroxybenzoic and then to 3-octaprenyl-4-hydroxybenzoate, which are the first two steps of the biosynthesis of ubiquinone [17]. Ubiquinone is not only an essential component of the aerobic respiratory chain, but functions in the reduced form (ubiquinol) as an antioxidant, significantly reducing 


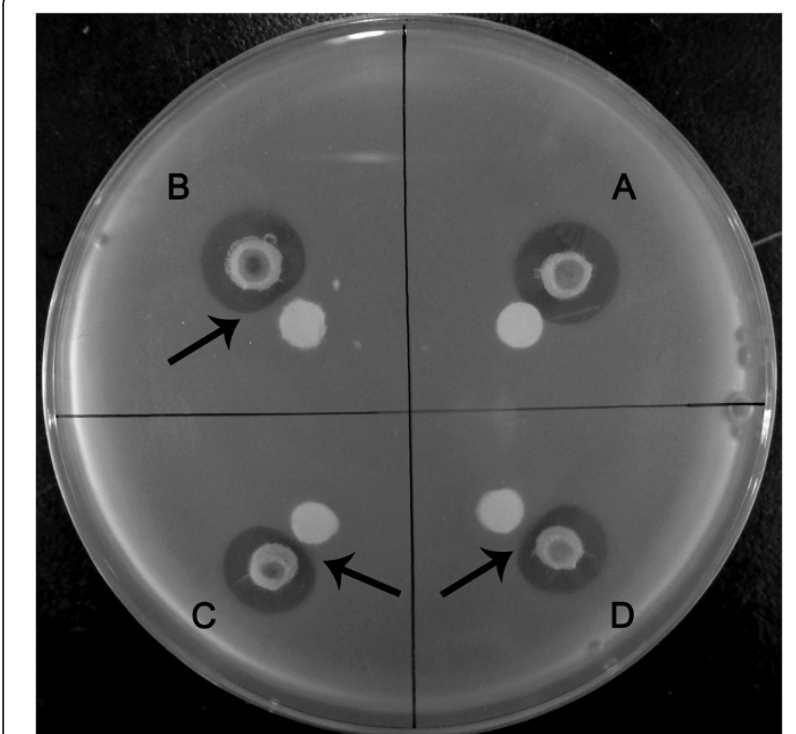

Figure 3 Catalase inhibition of the antibacterial effect of extracellular proteins against Vibrio anguillarum. A, disk with distilled water; $\mathbf{B}$, disk with $0.1 \mathrm{mg}$ of catalase; $\mathbf{C}$, disk with $0.2 \mathrm{mg}$ of catalase; $\mathbf{D}$, disk with $0.5 \mathrm{mg}$ of catalase.

oxidative stress, as for example generated by hydrogen peroxide [18]. We also identified all other genes in JG1 that are necessary for ubiquinone production.

For the biosynthesis of trans-cinnamic acid, two biosynthetic pathways can be taken into consideration: it can be derived from tyrosine by tyrosine ammonia lyase (TAL) directly [19], or cinnamic acid could get hydroxylated after its formation by phenylalanine ammonia lyase (PAL) [20]. Lyase-catalyzed reductive deamination releases ammonia thus forming the trans double bond found in the compound. This reaction sequence has been confirmed in Streptomyces maritimus [21] and Rhodobacter capsulatus [22]. However, in the genomic data of $P$. flavipulchra JG1 only two homologs of TAL (FaGL3502 and FaGL4409) can be identified. Therefore, this compound is most probably derived from tyrosine in JG1. trans-cinnamic acid is often found in plants. It has been used to augment the activity of various antibiotics against Mycobacterium avium [23] and exhibited synergistic effects with several anti-tuberculosis drugs active against $M$. tuberculosis [24]. In contrast to its wide occurrence in plants, trans-cinnamic acid is not very common in bacteria and only few reports on the biochemical characterization of TAL have been published [19].

Compound 6-bromoindolyl-3-acetic acid was shown to exhibit the strongest antibacterial activity among the small molecular compounds so far isolated from $P$. flavipulchra JG1. The metabolite can be formed by halogenation of tryptophan followed by oxidative deamination and decarboxylation. The indole-3-acetic acid (IAA) portion can be formed in bacteria following several IAA biosynthesis pathways, of which a single bacterial strain sometimes contains more than one [25]. IAA can be converted from indole-3-acetamide and indole-3-acetaldehyde via the indole-3-pyruvic acid pathway or the tryptamine pathway in JG1. The key enzymes involved in both of these pathways are present in the genome of JG1, including amino acid monooxygenase, indoleacetamide hydrolase, indole-3-pyruvate decarboxylase and monoamine oxidase. However, the tryptophan aminotransferase which transforms tryptophan into indole-3-pyruvate has not been detected in JG1. Alternatively, the reaction could be mediated by an L-amino acid oxidase and the PfaP protein might be a candidate. In addition, genes encoding tryptophan halogenases are found in the genome of JG1, one of which (FaGL1050) is located just downstream of $p f a P$. A tryptophan halogenase would catalyze bromination of tryptophan, which subsequently would be converted into 6-bromoindolyl-3-acetic acid via one of the above mentioned biosynthetic pathways (Figure 4). The PfaP protein, which is considered as a multifunctional protein, might be the most important antibacterial factor of $P$. flavipulchra thus far identified, as it also might play a role in the biosynthesis of 6bromoindolyl-3-acetic acid.

To assess the potential of P. flavipulchra JG1 to produce secondary metabolites that have not yet been isolated and chemically characterized, we analyzed its genome using antiSMASH [26]. This allowed for the identification of four different bacteriocin-type gene clusters, lantipeptide biosynthesis genes, four type I polyketide/non-ribosomal peptide (PKS/NRPS) hybrid clusters, three NRPS gene clusters (Additional file 2: Table S1), as well as a high number of further NRPSrelated peptidyl-carrier proteins (12), condensation (11), adenylation (12), epimerization (3), and thioesterase (2) domains distributed in the smaller scaffolds derived from our sequencing efforts. This strain thus appears to harbor tremendous potential to produce a large variety of peptide-based secondary metabolites. It is interesting to note that in terms of polyketide biosynthesis only type I PKS genes have been identified, all of these being part of larger NRPS biosynthetic loci. Among the biosynthetic gene clusters predicted by antiSMASH analysis, we identified a set of genes that can be correlated to polycyclic tetramic acid containing macrolactams (PTMs). This class of secondary metabolite gene clusters was recently found to be widespread among bacterial species and encodes the assembly of structurally complex, polycyclic secondary metabolites with a broad range of biological activities [27]. Given the apparent genetic potential of $P$. flavipulchra JG1 to produce complex bioactive natural products, further studies to chemically unlock its secondary metabolite profile seem to be extremely promising. 


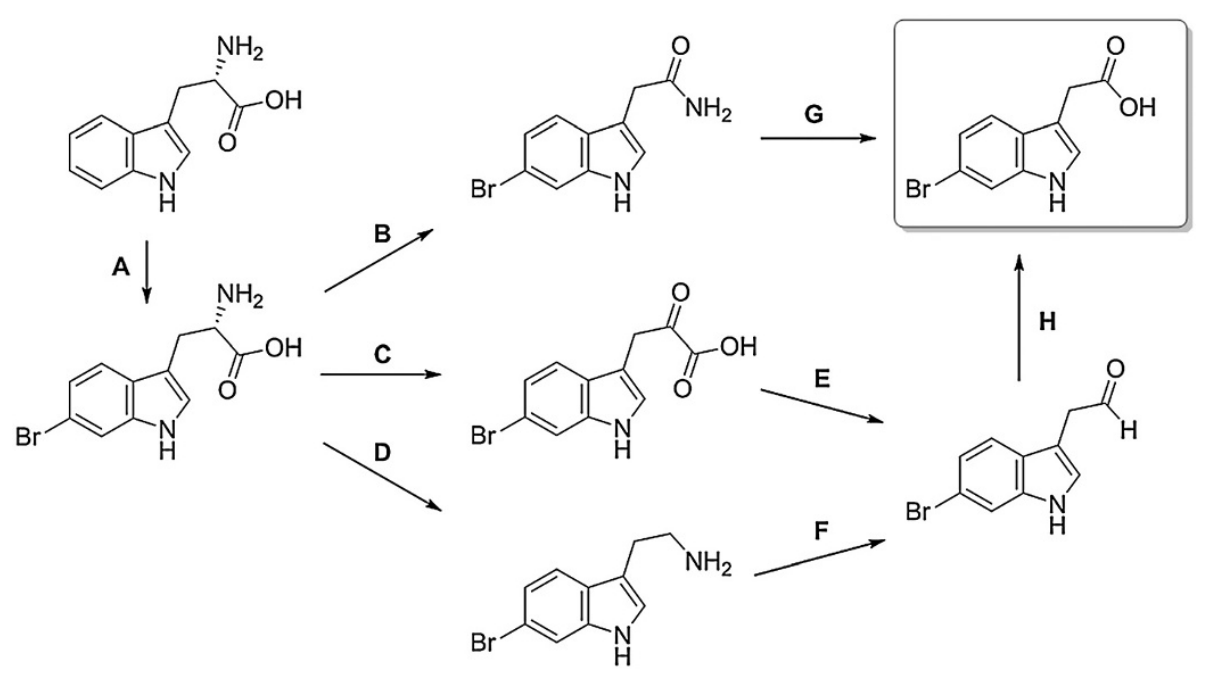

Figure 4 The biosynthesis pathways of 6-bromoindolyl-3-acetic acid in JG1. A, tryptophan halogenase; B, amino acid monooxygenase; C, L-amino acid oxidase; $\mathbf{D}$, tryptophan decarboxylase; E, indole-3-pyruvate decarboxylase; $\mathbf{F}$, monoamine oxidase; $\mathbf{G}$, indoleacetamide hydrolase; $\mathbf{H}$, aldehyde dehydrogenase.

Besides these secondary metabolites potential, $P$. flavipulchra JG1 was shown great chitinase activity on chitin agar. Chitinase can catalyze the hydrolysis of $\beta-1$, 4 glycosidic bonds linking the $N$-acetylglucosamine subunits of chitin in a variety of organisms, and the enzyme has been found in all sorts of organisms including bacteria, fungi, plants and animals [28,29]. Moreover, chitinase can be classified into two families as glycoside hydrolases (GH) 18 and 19 based on the amino acid sequence similarity [30,31]. P. flavipulchra JG1 harbored 5 genes (faGL1724, faGL2271, faGL2273, faGL2526 and faGL4793) coding for this enzyme, four of which belong to GH18. In the genomes of $P$. tunicata D2 and Pseudoalteromonas strains SM9913, by comparison, only two genes are annotated as chitinases, while no genes encoding this enzyme are found in $P$. haloplanktis TAC125 and P. atlantica T6c. Notably, chitinase has been confirmed to show antifungal activities in $B$. cereus [32] and Streptomyces sp. MG3 [28] as chitin is a component of the cell walls of fungi. Two extracellular chitinases purified from Pseudomonas aeruginosa K-187 also showed lysozyme activities against both gram-positive and gramnegative bacteria [33]. The chitinases identified in JG1 can thus be considered as antibacterial/antifungal proteins with a different antagonizing mechanism.

\section{Quorum sensing and quorum quenching}

Quorum sensing is a process that has been developed by bacteria to allow communication with each other and to facilitate monitoring of their cell density by measuring the concentration of small secreted signal molecules, such as acyl homoserine lactone (AHL) [34]. Diverse functions of bacteria are regulated via quorum sensing systems, including antibiotic biosynthesis, motility patterns and expression of many virulence-related genes in some animal and plant pathogens [35]. The quorum sensing regulatory system QseB/QseC detected in the genome of JG1 is organized in an operon with qseB encoding the response regulator and qse $C$ the sensor histidine kinase. QseB/QseC belongs to two-component signal transduction systems which enable bacteria to sense, respond, and adapt to changes in their environment or in their intracellular state [36]. However, the major genes encoding autoinducers responsible for quorum sensing have not been detected in JG1. The system may thus only regulate genes encoding the expression and assembly of flagella, motility and chemotaxis in JG1, as a protein responsible for biogenesis of type IV pilus is just downstream to the QseB/QseC system.

Quorum quenching (QQ) describes all processes that interfere with quorum sensing [37]. QQ enzymes, including AHL lactonase, acylase, oxidase and reductase, have been discovered in many bacterial genuses, such as Bacillus, Rhodococcus, Pseudomonas and Ralstonia [38]. Extra-and intra-cellular products of strain JG1 were shown to degrade long-chain AHLs, such as C10-HSL, C12-HSL, C14-HSL, 3-oxo-C12-HSL and 3-oxo-C14HSL, but not short-chain AHLs (shorter than 10 carbons in the acyl chain), and the enzymatic activity could not be reversed by acidification. This suggested that there might be genes encoding AHL acylases in the genome of strain JG1. Two genes of JG1 (FaGL1422 and FaGL2554) encoding penicillin acylase show high homology with PvdQ [39] and QuiP [40], which in Pseudomonas aeruginosa PAO1 were demonstrated to degrade long chain AHLs. Moreover, QuiP could utilize long chain 
AHLs as sole sources of carbon and energy. The catalytically active serine residue of $\mathrm{N}$-terminal nucleophilic hydrolases, as found in the functionally verified AHL acylases PvdQ and QuiP, is present in both homologs found in JG1. These potential AHL acylases therefore equip strain JG1 with another mechanism to reduce the detrimental effects of pathogens and prevent or limit the impact of bacterial diseases in rearing animals.

\section{Acquisition of phosphorus}

Efficient uptake of phosphorus is important for marine microorganisms due to the low phosphorus level in the marine environment. The phosphate input in the metabolism of P. flavipulchra JG1 is controlled by the counterparts of PhoB/PhoR and PhoU [41] as well as several phosphate transport systems (PstABC and PstS) [42] and inorganic phosphate (Pi) transport systems (Pit) [43]. The Pst system, which is derepressed under conditions of Pi starvation, also regulates synthesis of alkaline phosphatase, a periplasmic protein produced in greatest quantity during Pi starvation [44]. Homologs to the two component regulatory system PhoR/PhoB found in JG1 are responsible to control phosphate starvation and the system may also serve as a general transduction system for the expression of genes involved in secondary metabolism. Significantly, Pi starvation may stimulate bacteria to produce various secondary metabolites. For example, antibiotics biosynthesis in Streptomyces lividans is negatively regulated by phosphate via the PhoR/PhoB system [45]. JG1 might therefore up-regulate the production of defensive primary and secondary metabolites in the oligotrophic marine environment, thereby even more efficiently combating competing (micro) organisms.

\section{General stress response}

As the natural habitats of bacteria are constantly subjected to deleterious and fluctuating conditions that can be harmful, bacteria all have evolved their abilities to sense and respond to these environmental changes. RpoS, RelA, universal stress protein A, starvation stringent protein (SspB) and phage shock proteins (PspA-E) that are involved in controlling carbon and nutrient starvation [8] are present in P. flavipulchra JG1. Furthermore, the genome of JG1 also encodes for a large number of proteins involved in oxidative stress and metal homeostasis.

Besides three antioxidative proteins of the AhpC/Tsa family, including catalase, superoxide dismutase and an alkyl hydroperoxide reductase, JG1 has two proteins of the AhpF/TR family identified as AhpF and thioredoxin reductase [46]. These antioxidant enzymes protect JG1 against peroxide derived DNA damage as well as oxidative membrane or lipids destruction. Key regulators of the oxidative stress response are also present (such as
SoxR). Unlike P. tunicata D2 and P. haloplanktis TAC125 [13], the ubiquitous molybdopterin metabolism might be present, since dinucleotide-utilizing enzymes involved in molybdopterin biosynthesis can be found in JG1. However, genes coding for enzymes using cofactors, such as xanthine oxidase, biotin sulfoxide reductase [47], have not been detected. Five putative dioxygenases (FaGL2063, FaGL3237, FaGL2992, FaGL1118 and FaGL4206) might also help JG1 to protect its metabolism against oxidative stress.

Several genes involved in heavy metal detoxification were discovered in the genome of JG1, including periplasmic divalent cation tolerance protein (CutA) and copper homeostasis protein (CutC) [48]. Experiments proved that intracellular copper accumulation in E. coli could increase without cutA [49]. Moreover, cutA affects not only copper tolerance but also tolerance levels to zinc, nickel, cobalt and cadmium.

\section{Motility and secretion}

Flagellum formation is an important response to environmental stress. The swimming motility of $P$. flavipulchra JG1 observed by swimming plate method showed a distinct motility halo (Figure 5A), while the swarming ability was not detected (Figure 5B). Genes for synthesis of flagellum are present in P. flavipulchra JG1, and the resulting polar flagellum is visible under the transmission electron microscope (Figure 5C). Thirty three genes are involved in flagellar assembly in the P. flavipulchra JG1 genome. The class III flagellar operons which are controlled negatively by flgM are present [50]. FlgM is an inhibitory factor to sense the integrity of the bacterial flagellar structure and regulate flagellar synthesis by binding the transcription factor $\sigma^{28}$ [51]. However, the transcriptional activators FlhC and FlhD protein [52] were missing. FlhD alone also regulates the cell division rate, limiting cell division of bacterial communities entering the stationary phase, as shown for E. coli [53]. The deletion of FlhD may help JG1 to maintain a stable division rate to more quickly respond to environmental changes thus giving competitive advantages.

P. flavipulchra JG1 possesses three gene clusters for the biosynthesis of type IV pili including PilM/N/O/P/Q and PilF which interact to allow pilus assembly to occur, and also PilA, which is the most important component of type IV pili [54]. Pili mediate attachment to both living and artificial surfaces and are involved in bacteriophage adsorption, DNA uptake, biofilm initiation and development, and twitching motility [55]. One of the pili gene clusters and its upstream region is highly conserved in P. tunicata, P. haloplanktis and Pseudoalteromonas sp. TW-7 and SM9913 contains homologs to the two component response regulator system algZ/algR, which is involved in the regulation of alginate synthesis and pili- 


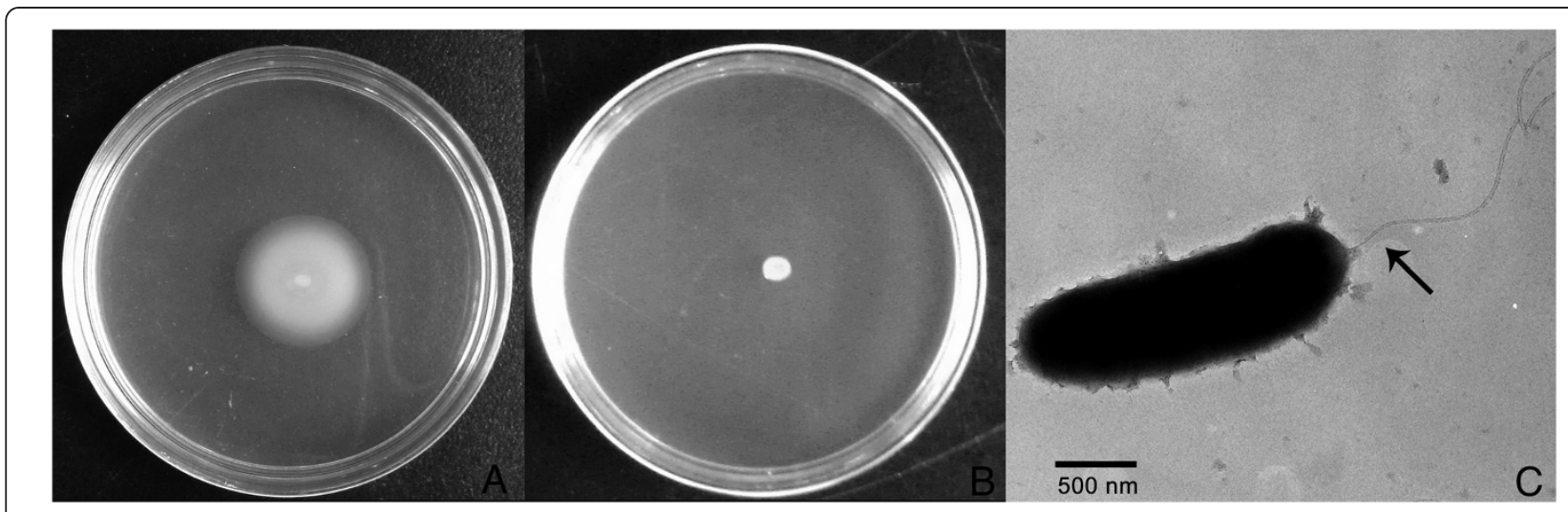

Figure 5 Swimming and swarming motility and transmission electron microscopy of $P$. flavipulchra JG1. (A) Distinct motility halo observed in the swimming plate; (B) No swarming ability observed in the swarming plate. (C) Transmission electron microscopy of P. flavipulchra JG1. The arrow indicated the polar flagellum.

mediated, twitching motility in Pseudomonas aeruginosa [56]. The alginate biosynthesis cluster is absent in JG1 as in $P$. tunicata, therefore, this regulatory system may only play a role in the expression of the pili cluster. With these appendages involved in motility, JG1 might be able to rapidly respond to environmental stresses and exceed other microorganisms.

There are several secretion systems functional in JG1, such as type I, II and VI secretion system, as well as the TAT and Sec-SRP export systems. In contrast, type III secretion system is absent. There are 15 genes involved in the type VI secretion system (T6SS). Previous studies indicated that many of these T6SS-containing bacteria are known pathogens and T6SS have been experimentally shown to play a role in virulence in several cases [57]. However, recent studies suggest that T6SS may limit bacterial replication or virulence and instead be used for intraspecies microbial cooperation, such as mediating antagonistic interactions between bacteria [58]. T6SS may be one of antibacterial mechanisms of JG1 that allows the bacterium inhibiting other microorganisms and affecting bacterial-host interactions.

\section{Drugs resistance and transport}

Microorganisms have developed various ways to resist the toxic effects of antibiotics and other drugs $[59,60]$. JG1 was shown excellent resistant activities to several common antibiotics such as penicillin, kanamycin, cephalosporin, tetracycline and chloramphenicol. One of the mechanisms may be enzymes that inactivate antibiotics by hydrolysis or the formation of inactive derivatives [61]. Penicillin metabolism in JG1 could be catalyzed by penicillin amidase yielding 6-aminopenicillanic acid, and by beta-lactamase (penicillinase) to give penicilloic acid. The penicillin amidase can also be found in TAC125, but not in other compared Pseudoalteromonas strains. JG1 also possesses D-amino-acid oxidase to convert cephalosporin C into (7R)-7-(5-carboxy-5-oxopentanoyl)aminocephalosporinate, consequently disarming this bactericide.

A second mechanism of antibiotic resistance is the inhibition of drug entry into the cell. The low permeability of the outer membrane of gram-negative bacteria could reduce drug diffusion across the cell envelope [62]. However, these barriers cannot entirely prevent all drugs from exerting their toxic action once they have entered the cell. The active efflux of drugs is thus essential to ensure the survival of the cell $[63,64]$. JG1 possesses many genes involved in defense mechanisms due to $\mathrm{ABC}$-type antimicrobial peptide transport system (34 genes), ABCtype multidrug transport system (16 genes), cation/ multidrug efflux pump (12 genes) and $\mathrm{Na}^{+}$-driven multidrug efflux pump (7 genes). The ABC-type transport systems have genes encoding ATPase and permease components. These multidrug transporters recognize lipophilic drugs by their physic-chemical properties that allow them to intercalate into the lipid bilayer, and transport these agents from the lipid bilayer to the exterior. The five small molecular compounds with antibacterial activity produced by JG1 were all lipophilic substances and multidrug transporters could reduce the intracellular accumulation of these compounds and protect the bacterium from the toxic effect of these bacteriostats. The transporters mediate the excretion of specific antibiotics in Streptomyces strains also dedicated to ensure self-resistance to the antibiotics that they produce [65].

\section{Conclusions}

The genome of $P$. flavipulchra JG1 unveils significant genetic advantages against other microorganisms, encoding antimicrobial agents as well as abilities to adapt to various adverse environments. The antibacterial protein $\mathrm{PfaP}$ not only catalytically produces hydrogen peroxide 
as a bacteriostat but likely also participates in the biosynthesis of small molecular antibacterial compound (6bromoindolyl-3-acetic acid). Both the macromolecule and small molecules contribute to the antibacterial activities of JG1. Besides these already identified chemical structures produced by strain JG1, a large number of peptide-based secondary metabolites encoded in the genome still awaits discovery. The identification of various antimicrobial enzymes enriches the antagonistic mechanisms of $P$. flavipulchra JG1 and could serve as therapeutic strategies against aquaculture pathogens. Furthermore, JG1 also evolves a range of mechanisms adapting the adverse marine environment or multidrug rearing conditions. The analysis of the genome of $P$. flavipulchra JG1 presented here provides a better understanding of its competitive properties and also an extensive application prospect.

\section{Methods}

\section{Bacterial growth and DNA extraction}

P. flavipulchra JG1 was isolated from rearing water of healthy turbot (Scophthalmus maximus) in Qingdao, China and was routinely grown on marine agar 2216 (MA; Difco) at $28^{\circ} \mathrm{C}$. Genomic DNA was extracted from $5 \mathrm{ml}$ overnight culture by standard methods [66]. Genomic DNA was quantified on $1 \%$ agarose gel stained with ethidium bromide and assessed spectrophotometrically.

The biosensors Chromobacterium violaceum CV026 and VIR24 were used to detect the short-chain (C4-C8) and long-chain (C8-C14) acyl homoserine lactones (AHLs), respectively. Both of them were grown on Luria-Bertani (LB) agar at $28^{\circ} \mathrm{C}$.

\section{Genome sequencing, annotation and analysis}

The genome sequence of P. flavipulchra JG1 was determined using the Illumina HiSeq2000 with a $500 \mathrm{bp}$ paired-end library and achieved about $600 \mathrm{Mb}$ data with 111.9-fold coverage. The reads were assembled using SOAPdenovo assembler software [67] subsequently. A total of 122 contigs ranging from 128 bp to $264447 \mathrm{bp}$ (the N50 and N90 contig sizes were $107608 \mathrm{bp}$ and 34 $132 \mathrm{bp}$, respectively) were obtained and combined into 61 scaffolds ranging from $500 \mathrm{bp}$ to $879239 \mathrm{bp}$ (the N50 and N90 contig sizes were $338061 \mathrm{bp}$ and 74731 $\mathrm{bp}$, respectively). Putative protein-encoding genes were identified with GLIMMER [68], transposons were predicted with Repeat Masker and Repeat Protein Masker [69], and tandem repeat sequences were identified through Tandem Repeat Finder [70]. Annotation was performed with BLASTALL 2.2.21 [71] searching against protein databases KEGG (Kyoto encyclopedia of genes and genomes; http://www.genome.jp/kegg/) [72], COG (http://www.ncbi.nlm.nih.gov/COG/) [73], SwissProt and TrEMBL (http://www.uniprot.org/) and NR (NCBI non- redundant database; http://www.ncbi.nlm.nih.gov/RefSeq/) [74]. The criteria used to assign function to a CDS were a minimum cutoff of $30 \%$ identity and at least four best hits among the COG, KEGG, NR, SwissProt or TrEMBL protein databases. Phylogenetic analysis was performed by alignment of sequences using Clustal $\mathrm{W}$ [75] and neighborjoining trees were generated with 1000 bootstraps. The prediction of signal peptides (SP) was performed using SignalP v 4.1 [76]. The conserved domains were predicted using Conserved Domain Database of NCBI [77].

\section{Nucleotide sequence accession numbers}

This Whole Genome Shotgun project has been deposited at DDBJ/EMBL/GenBank under the accession AJMP00000000. The version described in this paper is the first version, AJMP01000000.

\section{Comparative genomics}

According to the result of phylogenetic analysis, the genome sequences of $P$. tunicata D2 and P. haloplanktis TAC125 were retrieved from NCBI. Proteins from $P$. flavipulchra JG1 were compared with those of D2 and TAC125, using BLASTP with an E-value cutoff of 1e-5. Orthologous proteins are defined as reciprocal best hit proteins with a minimum $40 \%$ identity and $70 \%$ of the length of the query protein, calculated by the BLAST algorithm. Proteins without orthologs are considered to be specific proteins. The COG function category was analyzed by searching all predicted proteins against the COG database on the basis of the BLASTP.

\section{Catalase effect on the antibacterial activity}

An $8 \mathrm{~mm}$ disc loaded with $0.1 \mathrm{mg}, 0.2 \mathrm{mg}$ and $0.5 \mathrm{mg}$ of catalase in $10 \mu \mathrm{l}$ distilled water was used and placed neighboring to the circular wells loaded with $20 \mu \mathrm{l}$ extracellular proteins of $P$. flavipulchra JG1, respectively [14]. The catalase inhibition of antibacterial effect was observed by the eclipse of inhibition areas.

\section{AHLs degradation bioassay}

C6 to C14-HSL and 3-oxo-C6 to 3-oxo-C14-HSL were used for evaluating the AHL degradation activity of $P$. flavipulchra JG1. Briefly, extra- and intra-cellular products of strain JG1 were mixed with different acyl chains of AHLs, the final concentrations of these AHLs were 1 $\mu \mathrm{M}$ for C10-HSL, C12-HSL and C14-HSL, and $0.1 \mu \mathrm{M}$ for the rest AHLs. The mixtures were incubated at $28^{\circ} \mathrm{C}$ for 24 hours and the residual AHLs were detected by Chromobacterium violaceum CV026 and VIR24 plate assay [78].

\section{Electron microscopy and motility assay}

An overnight culture of strain JG1 was negatively stained with $1 \%$ phosphotungstic acid $(\mathrm{pH} 7.4)$ on a Formvar 
carbon-coated grid and observed with a transmission electron microscope (TEM-1200EX, Japan) [79]. Swimming and swarming motilities were evaluated by point inoculating JG1 on MA plates containing $0.3 \%$ and $0.5 \%$ agar, respectively. The plates were analyzed after incubation at $28^{\circ} \mathrm{C}$ for about $24 \mathrm{~h}$. The experiment was performed in triplicate.

\section{Chitinase activity and antibiotic resistance}

The chitinase activity of P. flavipulchra JG1 were observed using chitin agar following the method described by Hsu et al. [80]. Resistance to antibiotics of strain JG1 was investigated by the agar diffusion method using the filter discs containing different antibiotics. Briefly, 100 $\mu \mathrm{l}$ aliquots of overnight broth culture of JG1 were spread onto the surface of MA plates, and different antibiotic discs were placed on the target plates, respectively, which were then incubated at $28^{\circ} \mathrm{C}$ for $24 \mathrm{~h}$. Inhibition zones of the antibiotics-containing discs were observed.

\section{Additional files}

Additional file 1: Figure S1. Gene percentages assigned to all the COG categories of the orthologous and specific genes in P. flavipulchra JG1, P. tunicata D2 and P. haloplanktis TAC125. (A-C) Abundances of specific genes in P. flavipulchra JG1, P. tunicata D2 and $P$. haloplanktis TAC125 assigned to the COG categories. (D) Orthologous genes among these three genomes assigned to the COG categories. COG functional categories are described in Figure 2.

Additional file 2: Table S1. Genes within the gene clusters involved in secondary metabolites synthesis.

\section{Competing interests}

The authors declare that they have no competing interests.

\section{Authors' contributions}

$\mathrm{X}-\mathrm{HZ}$ and $\mathrm{XS}$ designed and oversighted the study. MY and KT performed the laboratory work, analyzed the data and drafted the manuscript, JL and TAMG analyzed the data. All authors read and approved the final manuscript.

\section{Acknowledgements}

We thank Dr. Robert J.C. McLean (Texas State University, USA) for biosensors C. violaceum CV026 and Dr. Tomohiro Morohoshi (Utusnomiya University, Japan) for C. violaceum VIR24. This work was supported by grants from the International Science and Technology Cooperation Programme of China (No. 2012DFG31990) and the National Natural Science Foundation of China (No. 40876067). Funding of the work in the laboratory of TAMG was provided by the DFG (GU 1233/2-1).

\section{Author details}

'College of Marine Life Sciences, Ocean University of China, Qingdao 266003, China. ${ }^{2}$ Kekulé-Institute of Organic Chemistry and Biochemistry, University of Bonn, Gerhard-Domagk-Straße 1, Bonn 53121, Germany. ${ }^{3}$ Mailing address: College of Marine Life Sciences, Ocean University of China, 5 Yushan Road, Qingdao 266003, China.

Received: 1 July 2013 Accepted: 14 October 2013

Published: 16 October 2013

\section{References}

1. Gauthier G, Gauthier M, Christen R: Phylogenetic analysis of the genera Alteromonas, Shewanella, and Moritella using genes coding for smallsubunit rRNA sequences and division of the genus Alteromonas into two genera, Alteromonas (emended) and Pseudoalteromonas gen. nov., and proposal of twelve new species combinations. Int J Syst Bacteriol 1995, 45:755-761.

2. Hoyoux A, Jennes I, Dubois P, Genicot S, Dubail F, Francois JM, Baise E, Feller G, Gerday C: Cold-adapted beta-galactosidase from the Antarctic psychrophile Pseudoalteromonas haloplanktis. Appl Environ Microbiol 2001, 67:1529-1535.

3. Kobayashi T, Imada C, Hiraishi A, Tsujibo H, Miyamoto K, Inamori Y, Hamada $\mathrm{N}$, Watanabe E: Pseudoalteromonas sagamiensis sp. nov., a marine bacterium that produces protease inhibitors. Int J Syst Evol Microbiol 2003, 53:1807-1811.

4. Egan S, Holmstrom C, Kjelleberg S: Pseudoalteromonas ulvae sp. nov., a bacterium with antifouling activities isolated from the surface of a marine alga. Int J Syst Evol Microbiol 2001, 51:1499-1504.

5. Bowman JP: Bioactive compound synthetic capacity and ecological significance of marine bacterial genus Pseudoalteromonas. Mar Drugs 2007, 5:220-241.

6. James SG, Holmstrom C, Kjelleberg S: Purification and characterization of a novel antibacterial protein from the marine bacterium D2. Appl Environ Microbiol 1996, 62:2783-2788

7. Franks A, Haywood P, Holmstrom C, Egan S, Kjelleberg S, Kumar N: Isolation and structure elucidation of a novel yellow pigment from the marine bacterium Pseudoalteromonas tunicata. Molecules 2005, 10:1286-1291.

8. Thomas T, Evans FF, Schleheck D, Mai-Prochnow A, Burke C, Penesyan A Dalisay DS, Stelzer-Braid S, Saunders N, Johnson J, et al: Analysis of the Pseudoalteromonas tunicata genome reveals properties of a surfaceassociated life style in the marine environment. PLoS One 2008, 3:e3252.

9. Jin G, Wang S, Yu M, Yan S, Zhang X-H: Identification of a marine antagonistic strain JG1 and establishment of a polymerase chain reaction detection technique based on the gyrB gene. Aquac Res 2010, 41:1867-1874

10. Yu M, Wang J, Tang K, Shi X, Wang S, Zhu W-M, Zhang X-H: Purification and characterization of antibacterial compounds of Pseudoalteromonas flavipulchra JG1. Microbiology 2012, 158:835-842.

11. Yu M, Tang K, Shi X, Zhang X-H: Genome sequence of Pseudoalteromonas flavipulchra JG1, a marine antagonistic bacterium with abundant antimicrobial metabolites. J Bacteriol 2012, 194:3735.

12. Egan S, James $S$, Kjelleberg S: Identification and characterization of a putative transcriptional regulator controlling the expression of fouling inhibitors in Pseudoalteromonas tunicata. Appl Environ Microbiol 2002, 68:372-378.

13. Medigue C, Krin E, Pascal G, Barbe V, Bernsel A, Bertin PN, Cheung F, Cruveiller S, D'Amico S, Duilio A, et al: Coping with cold: the genome of the versatile marine Antarctica bacterium Pseudoalteromonas haloplanktis TAC125. Genome research 2005, 15:1325-1335.

14. Lucas-Elio P, Gomez D, Solano F, Sanchez-Amat A: The antimicrobial activity of marinocine, synthesized by Marinomonas mediterranea, is due to hydrogen peroxide generated by its lysine oxidase activity. J Bacteriol 2006, 188:2493-2501.

15. Faust A, Niefind K, Hummel W, Schomburg D: The structure of a bacterial L-amino acid oxidase from Rhodococcus opacus gives new evidence for the hydride mechanism for dehydrogenation. J Mol Bio/ 2007, 367:234-248.

16. Zaldivar J, Ingram LO: Effect of organic acids on the growth and fermentation of ethanologenic Escherichia coli LY01. Biotechnol Bioeng 1999, 66:203-210.

17. Nichols BP, Green JM: Cloning and sequencing of Escherichia coli ubiC and purification of chorismate lyase. J Bacterio/ 1992, 174:5309-5316.

18. Soballe B, Poole RK: Ubiquinone limits oxidative stress in Escherichia coli. Microbiology 2000, 146(Pt 4):787-796.

19. Watts KT, Mijts BN, Lee PC, Manning AJ, Schmidt-Dannert C: Discovery of a substrate selectivity switch in tyrosine ammonia-lyase, a member of the aromatic amino acid lyase family. Chem Biol 2006, 13:1317-1326.

20. Xiang L, Moore BS: Inactivation, complementation, and heterologous expression of enc $P$, a novel bacterial phenylalanine ammonia-lyase gene. J Biol Chem 2002, 277:32505-32509.

21. Xiang $L$, Moore BS: Biochemical characterization of a prokaryotic phenylalanine ammonia lyase. J Bacterio/ 2005, 187:4286-4289. 
22. Kyndt JA, Meyer TE, Cusanovich MA, Van Beeumen JJ: Characterization of a bacterial tyrosine ammonia lyase, a biosynthetic enzyme for the photoactive yellow protein. FEBS Lett 2002, 512:240-244.

23. Rastogi N, Goh KS, Horgen L, Barrow WW: Synergistic activities of antituberculous drugs with cerulenin and trans-cinnamic acid against Mycobacterium tuberculosis. FEMS Immunol Med Microbiol 1998, 21:149-157.

24. Chen YL, Huang ST, Sun FM, Chiang YL, Chiang CJ, Tsai CM, Weng CJ: Transformation of cinnamic acid from trans- to cis-form raises a notable bactericidal and synergistic activity against multiple-drug resistant Mycobacterium tuberculosis. Eur J Pharm Sci 2011, 43:188-194.

25. Patten CL, Glick BR: Bacterial biosynthesis of indole-3-acetic acid. Can J Microbiol 1996, 42:207-220.

26. Medema MH, Blin $K$, Cimermancic $P$, de Jager $V$, Zakrzewski $P$, Fischbach MA, Weber T, Takano E, Breitling R: antiSMASH: rapid identification, annotation and analysis of secondary metabolite biosynthesis gene clusters in bacterial and fungal genome sequences. Nucleic Acids Res 2011, 39:W339-W346.

27. Blodgett JA, Oh DC, Cao S, Currie CR, Kolter R, Clardy J: Common biosynthetic origins for polycyclic tetramate macrolactams from phylogenetically diverse bacteria. Proc Natl Acad Sci USA 2010, 107:11692-11697.

28. Hoster F, Schmitz JE, Daniel R: Enrichment of chitinolytic microorganisms: isolation and characterization of a chitinase exhibiting antifungal activity against phytopathogenic fungi from a novel Streptomyces strain. Appl Microbiol Biotechnol 2005, 66:434-442.

29. Lam YW, Wang HX, Ng TB: A robust cysteine-deficient chitinase-like antifungal protein from inner shoots of the edible chive Allium tuberosum. Biochem Biophys Res Commun 2000, 279:74-80.

30. Funkhouser JD, Aronson NN Jr: Chitinase family GH18: evolutionary insights from the genomic history of a diverse protein family. BMC Evol Biol 2007, 7:96

31. Kawase T, Saito A, Sato T, Kanai R, Fujii T, Nikaidou N, Miyashita K, Watanabe $\mathrm{T}$ : Distribution and phylogenetic analysis of family 19 chitinases in Actinobacteria. Appl Environ Microbiol 2004, 70:1135-1144.

32. Chang WT, Chen CS, Wang SL: An antifungal chitinase produced by Bacillus cereus with shrimp and crab shell powder as a carbon source. Curr Microbiol 2003, 47:102-108.

33. Wang SL, Chang WT: Purification and characterization of two bifunctional chitinases/lysozymes extracellularly produced by Pseudomonas aeruginosa K-187 in a shrimp and crab shell powder medium. Appl Environ Microbiol 1997, 63:380-386.

34. Galloway WR, Hodgkinson JT, Bowden SD, Welch M, Spring DR: Quorum sensing in Gram-negative bacteria: small-molecule modulation of $\mathrm{AHL}$ and Al-2 quorum sensing pathways. Chem Rev 2011, 111:28-67.

35. Williams P, Winzer K, Chan WC, Camara M: Look who's talking: communication and quorum sensing in the bacterial world. Philos Trans $R$ Soc Lond B Biol Sci 2007, 362:1119-1134.

36. Sperandio V, Torres AG, Kaper JB: Quorum sensing Escherichia coli regulators $B$ and $C$ (QseBC): a novel two-component regulatory system involved in the regulation of flagella and motility by quorum sensing in E. coli. Mol Microbiol 2002, 43:809-821.

37. Dong YH, Wang LH, Xu JL, Zhang HB, Zhang XF, Zhang LH: Quenching quorum-sensing-dependent bacterial infection by an $\mathrm{N}$-acyl homoserine lactonase. Nature 2001, 411:813-817.

38. Uroz S, Dessaux Y, Oger P: Quorum sensing and quorum quenching: the yin and yang of bacterial communication. Chembiochem 2009, 10:205-216.

39. Sio CF, Otten LG, Cool RH, Diggle SP, Braun PG, Bos R, Daykin M, Camara M, Williams P, Quax WJ: Quorum quenching by an N-acyl-homoserine lactone acylase from Pseudomonas aeruginosa PAO1. Infect Immun 2006, 74:1673-1682.

40. Huang JJ, Petersen A, Whiteley M, Leadbetter JR: Identification of QuiP, the product of gene PA1032, as the second acyl-homoserine lactone acylase of Pseudomonas aeruginosa PAO1. Appl Environ Microbiol 2006, 72:1190-1197.

41. Stock JB, Ninfa AJ, Stock AM: Protein phosphorylation and regulation of adaptive responses in bacteria. Microbiol Rev 1989, 53:450-490.

42. Crepin S, Chekabab SM, Le Bihan G, Bertrand N, Dozois CM, Harel J: The Pho regulon and the pathogenesis of Escherichia coli. Vet Microbiol 2011, 153:82-88.

43. Hsieh YJ, Wanner BL: Global regulation by the seven-component $\mathrm{Pi}$ signaling system. Curr Opin Microbiol 2010, 13:198-203.
44. Willsky GR, Malamy MH: Characterization of two genetically separable inorganic phosphate transport systems in Escherichia coli. J Bacteriol 1980, 144:356-365.

45. Sola-Landa A, Moura RS, Martin JF: The two-component PhoR-PhoP system controls both primary metabolism and secondary metabolite biosynthesis in Streptomyces lividans. Proc Natl Acad Sci USA 2003, 100:6133-6138.

46. Chae HZ, Robison K, Poole LB, Church G, Storz G, Rhee SG: Cloning and sequencing of thiol-specific antioxidant from mammalian brain: alkyl hydroperoxide reductase and thiol-specific antioxidant define a large family of antioxidant enzymes. Proc Natl Acad Sci USA 1994, 91:7017-7021.

47. Loschi L, Brokx S, Hills T, Zhang G, Bertero M, Lovering A, Weiner J, Strynadka N: Structural and biochemical identification of a novel bacterial oxidoreductase. J Biol Chem 2004, 279:50391-50400.

48. Cooksey DA: Copper uptake and resistance in bacteria. Mol Microbiol 1993, 7:1-5.

49. Fong ST, Camakaris J, Lee BT: Molecular genetics of a chromosomal locus involved in copper tolerance in Escherichia coli K-12. Mol Microbiol 1995, 15:1127-1137.

50. Chilcott GS, Hughes KT: Coupling of flagellar gene expression to flagellar assembly in Salmonella enterica serovar typhimurium and Escherichia coli. Microbiol Mol Biol Rev 2000, 64:694-708.

51. Gillen KL, Hughes KT: Molecular characterization of flgM, a gene encoding a negative regulator of flagellin synthesis in Salmonella typhimurium. J Bacteriol 1991, 173:6453-6459.

52. Liu X, Matsumura P: The FlhD/FlhC complex, a transcriptional activator of the Escherichia coli flagellar class II operons. J Bacteriol 1994, 176:7345-7351.

53. Pruss BM, Matsumura P: A regulator of the flagellar regulon of Escherichia coli, flhD, also affects cell division. J Bacteriol 1996, 178:668-674.

54. Spangenberg C, Fislage R, Sierralta W, Tummler B, Romling U: Comparison of type IV-pilin genes of Pseudomonas aeruginosa of various habitats has uncovered a novel unusual sequence. FEMS Microbiol Lett 1995, 125:265-273.

55. Craig L, Taylor RK, Pique ME, Adair BD, Arvai AS, Singh M, Lloyd SJ, Shin DS, Getzoff ED, Yeager $M$, et al: Type IV pilin structure and assembly: X-ray and EM analyses of Vibrio cholerae toxin-coregulated pilus and Pseudomonas aeruginosa PAK pilin. Mol Cell 2003, 11:1139-1150.

56. Baynham PJ, Ramsey DM, Gvozdyev BV, Cordonnier EM, Wozniak DJ: The Pseudomonas aeruginosa ribbon-helix-helix DNA-binding protein AlgZ (AmrZ) controls twitching motility and biogenesis of type IV pili. J Bacteriol 2006, 188:132-140.

57. Bingle LE, Bailey CM, Pallen MJ: Type VI secretion: a beginner's guide. Curr Opin Microbiol 2008, 11:3-8.

58. Jani AJ, Cotter PA: Type VI secretion: not just for pathogenesis anymore. Cell Host Microbe 2010, 8:2-6.

59. Hayes JD, Wolf CR: Molecular mechanisms of drug resistance. Biochem J 1990, 272:281-295.

60. Neu HC: The crisis in antibiotic resistance. Science 1992, 257:1064-1073.

61. Davies J: Inactivation of antibiotics and the dissemination of resistance genes. Science 1994, 264:375-382

62. Nikaido $\mathrm{H}$ : Molecular basis of bacterial outer membrane permeability revisited. Microbiol Mol Biol Rev 2003, 67:593-656.

63. Levy SB: Active efflux mechanisms for antimicrobial resistance. Antimicrob Agents Chemother 1992, 36:695-703.

64. Nikaido H: Prevention of drug access to bacterial targets: permeability barriers and active efflux. Science 1994, 264:382-388.

65. Linton KJ, Cooper HN, Hunter IS, Leadlay PF: An ABC-transporter from Streptomyces longisporoflavus confers resistance to the polyetherionophore antibiotic tetronasin. Mol Microbiol 1994, 11:777-785.

66. Ausubel FM, Brent R, Kingston RE, Moore DD, Seidman JG, Smith JA, Struhl K: Short protocols in molecular biology: a compendium of methods from current protocols in molecular biology. 3rd edition. New York, USA: Wiley; 1995.

67. Li R, Zhu H, Ruan J, Qian W, Fang X, Shi Z, Li Y, Li S, Shan G, Kristiansen K, et al: De novo assembly of human genomes with massively parallel short read sequencing. Genome research 2010, 20:265-272.

68. Delcher AL, Bratke KA, Powers EC, Salzberg SL: Identifying bacterial genes and endosymbiont DNA with Glimmer. Bioinformatics 2007, 23:673-679.

69. Tarailo-Graovac M, Chen N: Using RepeatMasker to identify repetitive elements in genomic sequences. Curr Protoc Bioinform 2009, 25:4.10.11-14.10.14.

70. Benson G: Tandem repeats finder: a program to analyze DNA sequences. Nucleic Acids Res 1999, 27:573-580. 
71. Altschul SF, Madden TL, Schaffer AA, Zhang J, Zhang Z, Miller W, Lipman DJ: Gapped BLAST and PSI-BLAST: a new generation of protein database search programs. Nucleic Acids Res 1997, 25:3389-3402.

72. Ogata H, Goto S, Sato K, Fujibuchi W, Bono H, Kanehisa M: KEGG: Kyoto Encyclopedia of Genes and Genomes. Nucleic Acids Res 1999, 27:29-34.

73. Tatusov RL, Koonin EV, Lipman DJ: A genomic perspective on protein families. Science 1997, 278:631-637.

74. Pruitt KD, Tatusova T, Maglott DR: NCBI reference sequences (RefSeq): a curated non-redundant sequence database of genomes, transcripts and proteins. Nucleic Acids Res 2007, 35:D61-D65.

75. Thompson JD, Higgins DG, Gibson TJ: CLUSTAL W: improving the sensitivity of progressive multiple sequence alignment through sequence weighting, position-specific gap penalties and weight matrix choice. Nucleic Acids Res 1994, 22:4673-4680.

76. Petersen TN, Brunak S, von Heijne G, Nielsen H: SignalP 4.0: discriminating signal peptides from transmembrane regions. Nat Methods 2011, 8:785-786.

77. Marchler-Bauer A, Lu S, Anderson JB, Chitsaz F, Derbyshire MK, DeWeeseScott C, Fong JH, Geer LY, Geer RC, Gonzales NR, et al: CDD: a Conserved Domain Database for the functional annotation of proteins. Nucleic Acids Res 2011, 39:D225-D229.

78. Someya N, Morohoshi T, Okano N, Otsu E, Usuki K, Sayama M, Sekiguchi H, Ikeda T, Ishida S: Distribution of $\mathrm{N}$-acylhomoserine lactone-producing fluorescent pseudomonads in the phyllosphere and rhizosphere of potato (Solanum tuberosum L.). Microbes Environ 2009, 24:305-314.

79. He Y, Xu T, Fossheim LE, Zhang XH: FliC, a flagellin protein, is essential for the growth and virulence of fish pathogen Edwardsiella tarda. PLoS One 2012, 7:e45070.

80. Hsu S, Lockwood J: Powdered chitin agar as a selective medium for enumeration of actinomycetes in water and soil. Appl Microbiol 1975, 29:422-426.

doi:10.1186/1471-2164-14-707

Cite this article as: Yu et al:: Genome analysis of Pseudoalteromonas

flavipulchra JG1 reveals various survival advantages in marine environment. BMC Genomics 2013 14:707.

\section{Submit your next manuscript to BioMed Central and take full advantage of:}

- Convenient online submission

- Thorough peer review

- No space constraints or color figure charges

- Immediate publication on acceptance

- Inclusion in PubMed, CAS, Scopus and Google Scholar

- Research which is freely available for redistribution 